Published in final edited form as:

J Pediatr Gastroenterol Nutr. 2017 May ; 64(5): 713-720. doi:10.1097/MPG.0000000000001335.

\title{
Use of Reticulocyte Hemoglobin Content in the Assessment of Iron Deficiency in Children with Inflammatory Bowel Disease
}

\author{
Sana Syed ${ }^{1,2}$, Subra Kugathasan ${ }^{1,2}$, Archana Kumar ${ }^{1}$, Jarod Prince ${ }^{1}$, Bess T. Schoen ${ }^{1,2}$, \\ Courtney McCracken ${ }^{1}$, Thomas R. Ziegler ${ }^{3}$, and Parminder S. Suchdev ${ }^{1,2}$
}

\begin{abstract}
Correspondence: Sana Syed, MD MSc, Emory University School of Medicine, Division of Pediatric Gastroenterology, Health Science Research Building (HSRB), 1760 Haygood Drive, W427, Atlanta, GA 30322, Tel: 404727 4542, Fax: 404727 4069, syedsana@gmail.com.
\end{abstract}

Conflict of Interest Statement for all authors:

SS: None relevant to this publication

SK: None relevant to this publication

AK: None relevant to this publication

JP: None relevant to this publication

BTS: None relevant to this publication

$\mathrm{CM}$ : None relevant to this publication

TRZ: None relevant to this publication

PSS: None relevant to this publication

Disclosures: No conflicts of interest.

Writing Assistance: None.

Author Contributions

Syed:

Substantial contributions to the conception or design of the work; and the acquisition, analysis AND Drafting the work or revising it critically for important intellectual content; AND Final approval of the version to be published; AND Agreement to be accountable for all aspects of the work in ensuring that questions related to the accuracy or integrity of any part of the work are appropriately investigated and resolved.

Kugathasan:

Substantial contributions to the conception or design of the work; or the acquisition, analysis, or interpretation of data for the work; AND revising the work critically for important intellectual content; AND Final approval of the version to be published; AND Agreement to be accountable for all aspects of the work in ensuring that questions related to the accuracy or integrity of any part of the work are appropriately investigated and resolved.

Kumar:

Substantial contributions to the acquisition, analysis, or interpretation of data for the work; AND revising it critically for important intellectual content; AND Final approval of the version to be published; AND Agreement to be accountable for all aspects of the work in ensuring that questions related to the accuracy or integrity of any part of the work are appropriately investigated and resolved. Prince:

Substantial contributions to the acquisition, analysis, or interpretation of data for the work; AND revising it critically for important intellectual content; AND Final approval of the version to be published; AND Agreement to be accountable for all aspects of the work in ensuring that questions related to the accuracy or integrity of any part of the work are appropriately investigated and resolved. Schoen:

Substantial contributions to the acquisition, analysis, or interpretation of data for the work; AND revising it critically for important intellectual content; AND Final approval of the version to be published; AND Agreement to be accountable for all aspects of the work in ensuring that questions related to the accuracy or integrity of any part of the work are appropriately investigated and resolved. McCracken:

Substantial contributions to the conception or design of the work AND Revising the work critically for important intellectual content; AND Final approval of the version to be published; AND Agreement to be accountable for all aspects of the work in ensuring that questions related to the accuracy or integrity of any part of the work are appropriately investigated and resolved.

Ziegler:

Substantial contributions to the conception or design of the work AND Revising the work critically for important intellectual content; AND Final approval of the version to be published; AND Agreement to be accountable for all aspects of the work in ensuring that questions related to the accuracy or integrity of any part of the work are appropriately investigated and resolved. Suchdev:

Substantial contributions to the conception or design of the work and acquisition, analysis, or interpretation of data for the work; AND revising it critically for important intellectual content; AND Final approval of the version to be published; AND Agreement to be accountable for all aspects of the work in ensuring that questions related to the accuracy or integrity of any part of the work are appropriately investigated and resolved. 
${ }^{1}$ Department of Pediatrics, Emory University School of Medicine, Atlanta, Georgia

${ }^{2}$ Children's Healthcare of Atlanta, Atlanta, Georgia

${ }^{3}$ Department of Medicine, Division of Endocrinology, Metabolism and Lipids, Emory University School of Medicine, Atlanta, GA

\section{Abstract}

BACKGROUND-Iron deficiency and anemia affect up to 50-75\% of inflammatory bowel disease (IBD) patients. Iron deficiency in IBD may be difficult to diagnose because of the effect of inflammation on iron status biomarkers. Thus, there is a need for better methods to accurately determine iron status in IBD.

OBJECTIVE-To investigate the association of inflammation with hemoglobin content of reticulocytes $(\mathrm{CHr})$ and the utility of $\mathrm{CHr}$ in comparison to standard iron biomarkers.

DESIGN/METHODS-We conducted a cross-sectional study of children with IBD. Iron biomarkers [CHr, ferritin, soluble transferrin receptor (sTfR), hepcidin, hemoglobin] were measured along with systemic biomarkers of inflammation [C-reactive protein (CRP), a 1-acid glycoprotein (AGP)]. Spearman correlations were used to evaluate the relationship of inflammation and iron biomarkers. The gold standard for iron deficiency was defined as inflammation-corrected ferritin $<15 \mu \mathrm{g} / \mathrm{L}$ or $\mathrm{sTfR}>8.3 \mathrm{mg} / \mathrm{L}$. Receiver operating characteristic (ROC) curves were used to estimate the prognostic values of all iron biomarkers to identify patients with iron deficiency.

RESULTS-We analyzed data in 62 children aged 5 to $<19$ years. Sixty-nine $\%$ of our subjects had Crohn's disease and 31\% had ulcerative colitis, of which $42 \%$ were females and 53\% African American. The prevalence of anemia was 32\%, of iron deficiency was 52\% using ferritin $<15$ $\mu \mathrm{g} / \mathrm{L}$ or $\mathrm{sTfR}>8.3 \mathrm{mg} / \mathrm{L}, 39 \%$ using RDW $>14.5 \%, 26 \%$ using BIS $<0 \mathrm{mg} / \mathrm{kg}$ body weight, $25 \%$ using $\mathrm{CHr}<28 \mathrm{pg}$ and $11 \%$ using $\mathrm{MCV}<75 \mathrm{fL} /$ cell. After correcting ferritin and sTfR levels for inflammation, the prevalence of iron deficiency was $68 \%$. CHr was correlated with CRP $\left(\mathrm{r}_{\mathrm{s}}-0.44\right.$, $\mathrm{p}<0.001)$ and AGP $\left(\mathrm{r}_{\mathrm{s}}-0.37, \mathrm{p}<0.05\right)$. The optimal prognostic value for inflammation-adjusted $\mathrm{CHr}$ to predict iron deficiency was $34 \mathrm{pg}$ (area under the ROC of 0.70 ), with $88 \%$ sensitivity and $30 \%$ specificity.

CONCLUSIONS-Iron deficiency and anemia are very common in this pediatric IBD cohort. All explored iron biomarkers, including $\mathrm{CHr}$, were affected by inflammation and should be adjusted. A single iron biomarker is unlikely to best predict iron deficiency in pediatric IBD. Iron intervention studies are needed to examine the response of iron biomarkers to iron supplementation in the setting of inflammation.

\section{Keywords}

iron deficiency; anemia; inflammation; Crohn's disease; ulcerative colitis; children

\section{INTRODUCTION}

Iron deficiency has significant negative morbidity with regards to quality of life and can lead to abnormal growth and inadequate cognitive development in children and adolescents (1). 
Prevalence estimates of iron deficiency and anemia in IBD have been reported as high as $50-75 \%$, with $\sim 50 \%$ being isolated iron deficiency with no anemia (2). Iron deficiency can be caused by an absolute iron deficiency (low iron stores, through a low dietary intake, malabsorption and/or gastrointestinal bleeding) or a functional iron deficiency (inflammation-associated cytokinemia with upregulated hepcidin production and resulting iron sequestration in enterocytes and macrophages and restricted erythropoiesis, resulting in anemia of chronic disease (3-5)). Micronutrient deficiencies often co-exist in the IBD population (1), including in particular iron and vitamin A deficiencies which have a role in microcytic anemia (6). Iron deficiency causes anemia which is associated with significant morbidity, including increased susceptibility to infection, reduced functionality of the musculoskeletal system, impaired intellectual functioning and subsequent impaired work capacity (1) (7). Anemia of chronic disease is anemia due to iron sequestration in patients with autoimmune disorders (such IBD), cancer, infections, and chronic kidney diseases (4). If left untreated, iron deficiency is associated with decreased quality of life with abnormal growth and poor cognitive development in children and adolescents (8) (5). Studies investigating the influence of vitamin A supplementation on iron status have shown simultaneous use of iron and vitamin A supplements seemed to be more effective to prevent iron deficiency anemia than the use of these micronutrients alone (6). Low vitamin A status has been documented both in $\mathrm{CD}$ and UC patients; furthermore, a relationship between disease severity and vitamin A deficiency has been observed among CD patients (9).

Iron status can be assessed in several ways with stainable bone marrow as the gold standard; however, most biomarkers are unreliable in the setting of inflammation as is often the case in patients with IBD, with or without acute disease exacerbations (7). The American Academy of Pediatrics and World Health Organization (WHO)/Centers of Disease Control and Prevention (CDC) guidelines for iron deficiency assessment in otherwise healthy children recommend the measurement of ferritin and/or soluble transferrin receptor (sTfR) along with assessment of inflammation by C-reactive protein (CRP) and/or a 1 acid glycoprotein (AGP) $(10,11)$. IBD guidelines recommend measurement of ferritin or transferrin saturation along with CRP as a measure of inflammation (12). Ferritin is an indicator of the storage iron content, but is itself an acute phase reactant and is therefore an unreliable measure of iron stores if inflammation is present (13). The challenge with using ferritin as an iron status biomarker in IBD is that the prevalence of iron deficiency is underestimated if the effect of inflammation is unaccounted for. Serum sTfR levels are increased in conditions of low iron availability for erythropoiesis, but in anemia of chronic disease are normal, due to the fact that transferrin receptor expression is negatively affected by inflammatory cytokines (14). Serum transferrin saturation is low in both iron deficiency and in anemia of chronic disease, so is not helpful in distinguishing between the two (14). Hepcidin has been prospectively demonstrated to negatively correlate with hemoglobin ( $\mathrm{Hgb}$ ) levels, supporting the hypothesis that IL-6-driven hepcidin production mediates anemia of chronic disease in patients with CD (15). There remains a pressing clinical need for better methods to accurately determine iron status in IBD to guide therapy that may improve clinical outcomes (16).

The hemoglobin concentration of reticulocytes $(\mathrm{CHr})(16)$ is a marker of iron status which has been shown to be an early and sensitive index of erythropoiesis (17), given the short 1- 
$2 \mathrm{~d}$ lifespan of the reticulocyte (18). $\mathrm{CHr}$ is considered to be an initial and reliable indicator of iron deficiency anemia, and unlike many other iron markers, is not affected by inflammation $(17,18)$. CHr is also an early indicator of the functional iron available for new red blood cell production over the previous 3-4 d (17). In IBD patients, there are no studies to date evaluating $\mathrm{CHr}$ as a reliable index to assess iron status or in response to iron supplementation therapy. We hypothesized that in pediatric patients with IBD, there is no association between $\mathrm{CHr}$ and markers of inflammation when compared to standard markers of iron status (ferritin and soluble transferrin receptor). Our aims were to: 1) determine the association of indices of iron status $(\mathrm{CHr}$, ferritin, soluble transferrin receptor) with inflammation in pediatric IBD patients; 2) describe the prevalence of iron deficiency, anemia and iron deficiency anemia in children with IBD; and 3) assess the sensitivity and specificity of $\mathrm{CHr}$ as a measure of iron status in this cohort.

\section{METHODS}

\section{Study Population and sample}

We performed a single center, cross-sectional study among children presenting to the pediatric IBD clinics, out-patient infusion clinics, emergency department and the in-patient gastroenterology service at the Children's Healthcare of Atlanta \& Emory University. Inclusion criteria were: (1) age of the participant 5-18 yrs; (2) confirmed diagnosis of IBD (CD, UD or IBD-unclassified) by a pediatric gastroenterologist. Patients meeting the following exclusion criteria were not considered eligible for the study: (1) patients with additional acute stress/inflammation such as those with history of any surgeries or infections within a one month period prior to study entry; (2) history of inherited blood disorders (thalassemia, sickle anemia or trait); (3) history of receiving any packed red blood cell infusion within 120d of study enrollment; (4) pregnant adolescents; (5) current mean corpuscular volume $(\mathrm{MCV})>100 \mathrm{fL} /$ cell (because of a presumption of vitamin B12 or folic acid deficiency which can also lead to anemia); (6) any iron supplementation. Patients were screened by review of all available prior clinical laboratory data within a one month time frame prior to their study visit to assess presence of inflammation and iron deficiency (Figure 1). All patients in our study population had disease surveillance labs (complete blood count, CRP) at routine intervals as part of their clinical care which we used as part of our screening. This allowed us to stratify our enrollment to target the following spectrum of patients: subjects with/without inflammation (CRP $>5 \mathrm{mg} / \mathrm{L}$ ) and with/without iron deficiency (MCV $<75 \mathrm{fL} /$ cell or RDW $>14.5 \%$ or anemia). Enrollment was done for a 6 month period from May 2014 to November 2014.

\section{Sample collection and laboratory assays}

Blood was collected from all subjects at the time of enrollment in a venous blood collection tube coated with EDTA (Beckman Dickinson). Whole blood samples of 3-5mL each were immediately sent for the following laboratory tests per standard clinical protocols: complete blood count (Siemens Advia 2120 and 120, Erlangen, Germany) and comprehensive metabolic panel (Siemens Vista 500, Erlangen, Germany). CHr (Siemens Advia, Erlangen, Germany) was also analyzed immediately on whole blood samples. Plasma was obtained by centrifuging the tube according to the manufacturer's specifications and then aliquoted and 
stored at $-80^{\circ} \mathrm{C}$. Frozen samples were shipped to the VitMin laboratory (Willstatt, Germany) for measurement of ferritin, CRP, AGP, sTfR and retinol binding protein (RBP) using a novel sandwich ELISA technique (19). Samples were also analyzed for hepcidin using the human hepcidin ELISA kit (TSZ Scientific, Waltham, MA) at Emory University as outlined by the kit's manufacturer. The average coefficient of variation of hepcidin among 11 subsamples was $18.8 \%$ (SD: 18.1, range: $1.1-64.6 \%$ ).

\section{Assessment of nutrition and health status}

The following thresholds were used to define abnormal values: 1) iron deficiency: ferritin < $15 \mathrm{mg} / \mathrm{L}$ (20), sTFR > $8.3 \mathrm{mg} / \mathrm{L}(21)$, body iron stores (BIS) $<0 \mathrm{mg} / \mathrm{kg}, \mathrm{CHr}<28 \mathrm{pg}$ (18); 2) vitamin A deficiency: retinol binding protein levels $<0.7 \mathrm{mmol} / \mathrm{L}$, vitamin A insufficiency: retinol binding protein levels 0.7 to $<1.0 \mathrm{mmol} / \mathrm{L}$ (22); 3) inflammation: CRP > 5mg/L (23), AGP $>1.0 \mathrm{~g} / \mathrm{L}$ (24). We calculated BIS for study participants using the formula based on the sTfR/ferritin ratio proposed by Cook et al (25), a measure considered to be reflective of iron status from decreased storage iron to functional tissue iron deficiency. Anemia was defined using the following thresholds for anemia per WHO guidelines (26); $\mathrm{Hb}<11.5 \mathrm{~g} / \mathrm{dL}$ for children aged 5-11.99 yrs, $\mathrm{Hb}<12.0 \mathrm{~g} / \mathrm{dL}$ for children aged 12-14.99 yrs, $\mathrm{Hb}<12.0 \mathrm{~g} / \mathrm{dL}$ for females aged $\geq 15.0 \mathrm{yrs}$ and $\mathrm{Hb}<13.0 \mathrm{~g} / \mathrm{dL}$ for males aged $\geq 15.0 \mathrm{yrs}$. The following data on each participant was collected: demographics (age, gender and self-reported race/ ethnicity), socio-economic status (SES) as measured by insurance status (state provided Medicaid or private insurance), clinical disease information (disease type, duration of disease, disease location, history of prior surgeries, disease activity, prior and current medical therapy). Age was categorized into two groups using a cut-off of 10.0 years - this was a biologically determined cut off for mean age of menarche in of US females using prior literature (27). Measures of anthropometrics included measurements of weight and height using standardized techniques by trained clinical nurses. Disease location was classified using the Paris classification for inflammatory bowel disease (28). Disease activity was assessed using the following indexes: Physician Global Assessment (PGA) score (29), abbreviated Pediatric Crohn's Disease Activity Index (AbbrPCDAI) (30) and Pediatric Ulcerative Colitis Activity (PUCAI) (31). The abbreviated PCDAI was used in our study as growth velocity information was not available to us - this index has been reported to be comparable to the PCDAI in recent literature (28-30).

\section{Statistical Methods}

Statistical analyses were performed using SAS 9.3 (SAS Institute Inc., Cary, NC). Statistical significance was set at a 2-sided alpha of 0.05. Descriptive statistics were presented as means (standard deviation, SD) and as 'n' (percent) for continuous and categorical outcomes, respectively. We used the WHO Child Growth Standards (WHO Anthro, Geneva, Switzerland) to calculate age and sex adjusted z-scores for anthropometric measurements. Further categorization of z-scores were as follows: stunting as a height-for-age $\mathrm{z}$-score of less than -2 , wasting as BMI for-age $z$-score of less than -2 , overweight as a BMI for-age $z$ score of greater than 2 and obesity as a BMI for-age z-score of greater than 3. Underweight was defined as weight-for-age z-score of less than -2 and this was calculated for children < 10 years of age due to availability of WHO reference standards only in this age group. All biomarkers were examined for normality using histograms, normal probability plots and 
using the Anderson-Darling test for normality. Correlations were calculated using Pearson or Spearman's correlation coefficient with associated $95 \%$ confidence. The receiver operating characteristic (ROC) curves and areas under the ROC curves (AUROC) were used to estimate the prognostic values (specificity and sensitivity) of $\mathrm{CHr}$ and other biomarkers. Cut-points for biomarkers were chosen to maximize sensitivity and specificity.

In the absence of the gold standard for iron deficiency as defined by Prussian blue staining of bone marrow iron stores, we used regression modeling to adjust ferritin and sTfR for inflammation as recently recommended by our group (32) and used a combination of multiple indicators to best estimate iron deficiency, as performed in the NHANES surveys in preschool-aged children $(33,34)$. Briefly, the regression correction approach uses linear regression to adjust a given iron biomarker by the level of AGP and/or CRP on a continuous scale. The first step in the regression correction approach was to natural logarithm (ln)transform each iron biomarker along with AGP and CRP concentrations to approximate normal distributions based on regression diagnostics. The least squares regression coefficients for AGP and/or CRP were obtained separately in a bivariate and multivariate model for each iron biomarker. The estimated slopes (i.e., regression coefficients) representing the relationship between AGP and/or CRP and the iron biomarker were then used to create an adjustment factor that could be added or subtracted to the biomarker based on the level of inflammation present. For sTfR, only AGP was used in the regression model based on the relationship we observed and prior literature suggesting that CRP and sTfR are not correlated $(35,36)$. Ferritin was regression-corrected for both AGP and CRP. A reference value was generated to define little or no inflammation by using the 10th percentile of AGP and/or CRP concentration in our dataset to avoid over adjusting the iron biomarkers. Thus, we defined iron deficiency as an inflammation-adjusted ferritin $<15 \mathrm{mg} / \mathrm{L}$ or an inflammation-adjusted sTFR $>8.3 \mathrm{mg} / \mathrm{L}$ and examined the sensitivity and specificity of other biomarkers to this standard.

\section{Access to Study Data}

All authors had access to the study data and have reviewed and approved this final manuscript.

\section{RESULTS}

\section{Demographics \& Race}

We analyzed data for 62 children aged 5 to $<19$ years. A total of $42 \%(n=26)$ were females, $10 \%(\mathrm{n}=7)$ were stunted or wasted and $10 \%(\mathrm{n}=7)$ were overweight. There were only seven children aged $<10$ years of whom none were underweight. Our population was primarily African American $(n=33,53 \%)$ with the remaining patients being mainly Caucasian $(n=25$, $40 \%$ ). Demographic characteristics are summarized in online supplementary Table S1.

\section{Clinical characteristics}

Our study population had predominately CD $(69 \%, n=43)$ with $31 \%(n=19)$ having UC. Average duration of disease was $3 \pm 3$ years, with the majority of patients having had no prior surgery $(n=44,71 \%)$. Classifying disease activity using PGA scores, most of our 
patients had either inactive or mild disease $(n=46,75 \%)$ with a small number of severely ill patients $(n=4,6 \%)$.

\section{Inflammation, Iron Deficiency, Anemia and vitamin A status}

Of the iron biomarkers measured, the following were normally distributed: $\mathrm{CHr}, \mathrm{MCV}, \mathrm{Hb}$, RBP; and the following were non-normally distributed: ferritin, sTfR, hepcidin, RDW, BIS. Online supplementary Table S2 outlines the descriptive summary of our iron biomarkers. We compared the mean values of $\mathrm{Hb}, \mathrm{RDW}, \mathrm{MCV}$, hepcidin and iron biomarkers adjusted for inflammation (CHr, ferritin, sTfR) in children with severe versus not severe (inactive/mild/ moderate) disease in our study (online supplementary Table S3) and found $\mathrm{Hb}$ and RDW with significant differences between the groups.

Table 1 summarizes the nutritional and inflammation status of the study. Nearly half of our subjects were inflamed (elevated CRP or AGP above cut points) and the prevalence of anemia was $32 \%(\mathrm{n}=20)$. Iron deficiency prevalence varied by biomarker used, with the range of prevalence (from low to high) as follows: $11 \%(\mathrm{n}=7)$ using $\mathrm{MCV}<75 \mathrm{fL} / \mathrm{cell}, 25 \%$ $(\mathrm{n}=15)$ using $\mathrm{CHr}<28 \mathrm{pg}, 26 \%$ using BIS $<0 \mathrm{mg} / \mathrm{kg}$ body weight, $39 \%$ ( $\mathrm{n}=24)$ using RDW $>14.5 \%$ and $52 \%(\mathrm{n}=32)$ using unadjusted ferritin $<15 \mu \mathrm{g} / \mathrm{L}$ or unadjusted sTfR $>$ $8.3 \mathrm{mg} / \mathrm{L}$. Using ferritin and sTfR, both adjusted for inflammation using regression, resulted in the highest iron deficiency prevalence estimate of $68 \%(n=42)$. Of the patients who were iron deficiency $(\mathrm{n}=42$, using ferritin $<15 \mu \mathrm{g} / \mathrm{L}$ or $\mathrm{sTfR}>8.3 \mathrm{mg} / \mathrm{L})$, half $(50 \%, \mathrm{n}=21)$ were inflamed (online supplementary Table S4). Prevalence of iron deficiency anemia (ferritin < $15 \mu \mathrm{g} / \mathrm{L}$ or $\mathrm{sTfR}>8.3 \mathrm{mg} / \mathrm{L}$, both adjusted for inflammation with $\mathrm{Hb}$ in anemic range) was $27 \%(\mathrm{n}=17)$. Of the patients who were anemic $(\mathrm{n}=20)$, approximately a quarter of patients, $65 \%(n=13)$ had duration of disease $>=2$ years and in iron deficiency patients $(n=42), 60 \%$ $(\mathrm{n}=25)$ had duration of disease $>=2$ years (online supplementary Tables S4 \&S5). Patients with $\mathrm{CD}$ had a higher prevalence of both anemia $(\mathrm{n}=15,75 \%)$ and iron deficiency $(\mathrm{n}=31$, $74 \%$ ) versus those with $\mathrm{UC}$ (anemia $\mathrm{n}=5,25 \%$; iron deficiency $\mathrm{n}=11,26 \%$ ) but this difference was not statistically significant. The prevalence of vitamin A deficiency was $3 \%$ $(\mathrm{n}=2)$ and vitamin A insufficiency was $13 \%(\mathrm{n}=8)$.

\section{Iron deficiency biomarkers and relationship with inflammation}

Table 2 summarizes the Spearman correlation coefficients relating biomarkers of inflammation to iron status indicators. Our findings were notable for a strong association with both biomarkers of systemic inflammation with $\mathrm{CHr}\left(\mathrm{CRP}, \mathrm{r}_{\mathrm{s}}=-0.44, \mathrm{CI}=-0.62\right.$ to $-0.21, \mathrm{P}=0.0003$ and AGP, $\mathrm{r}_{\mathrm{s}}=-0.37, \mathrm{CI}=-0.57$ to $\left.-0.13, \mathrm{P}=0.003\right)$, ferritin $\left(\mathrm{CRP}, \mathrm{r}_{\mathrm{s}}=\right.$ $0.27,95 \% \mathrm{CI}=0.02$ to $0.49, \mathrm{P}=0.034$ and $\mathrm{AGP}, \mathrm{r}_{\mathrm{s}}=0.36,95 \% \mathrm{CI}=0.12$ to $0.56, \mathrm{P}=0.004$ ), BIS (CRP, $r_{\mathrm{s}}=0.33,95 \% \mathrm{CI}=0.09$ to $0.54, \mathrm{P}=0.008$ and $\mathrm{AGP}, \mathrm{r}_{\mathrm{s}}=0.46,95 \% \mathrm{CI}=0.24$ to $0.64, \mathrm{P}<0.001)$ and $\mathrm{MCV}\left(\mathrm{CRP}, \mathrm{r}_{\mathrm{s}}=-0.41, \mathrm{CI}=-0.60\right.$ to $-0.18, \mathrm{P}<0.001$ and AGP, $\mathrm{r}_{\mathrm{s}}=$ $-0.32, \mathrm{CI}=-0.52$ to $-0.07, \mathrm{P}=0.011)$. sTfR was the only iron status biomarker which showed significant correlations only with AGP as a measure of inflammation: $\mathrm{sTfR}, \mathrm{r}_{\mathrm{s}}=$ $-0.32, \mathrm{CI}=-0.53$ to $-0.08, \mathrm{P}=0.010$. Hepcidin was strongly negatively correlated with $\mathrm{Hb}$ $\left(\mathrm{r}_{\mathrm{s}}=-0.50 ; \mathrm{CI}=-0.67\right.$ to $\left.-0.28 ; \mathrm{P}<0.0001\right)$. 


\section{Receiver operating characteristic (ROC) analysis}

Cutoff values for optimal sensitivity and specificity were obtained using ROC curves (Figure 2, Table 3). According to ROC curve, the optimal prognostic value for $\mathrm{CHr}$ to predict iron deficiency was $31 \mathrm{pg}$ with an AUROC of 0.72 with $81 \%$ sensitivity and $60 \%$ specificity (Table 3). Figure 2 summarizes prognostic values of other iron biomarkers (ferritin, reticulocyte hemoglobin concentration, sTfR, $\mathrm{Hb}$, red cell width, mean cell volume and hepcidin) according to ROC analysis. Adjusted ferritin had the highest area under the ROC with the optimal prognostic value to predict iron deficiency being $20 \mu \mathrm{g} / \mathrm{L}$ (sensitivity $79 \%$, specificity $90 \%$, AUROC 0.83 ). The poorest predictor of iron deficiency using the ROC was hepcidin (sensitivity $45 \%$, specificity $60 \%$, AUROC 0.54 ).

\section{DISCUSSION}

In this cross-sectional study, we evaluated the reticulocyte parameter $\mathrm{CHr}$ for diagnosis of iron deficiency in a population of children with IBD and demonstrated that $\mathrm{CHr}$ is affected by inflammation in children with IBD. Iron deficiency and anemia were common in this cohort of children with the prevalence of iron deficiency $68 \%$ and anemia $32 \%$ (iron deficiency anemia $27 \%$ ) after adjusting iron biomarkers data for inflammation. Interestingly, about $2 / 3$ rds of both anemic and iron deficiency patients had a duration of disease $>=2$ years. There was higher prevalence of anemia and iron deficiency in patients with CD (anemia $n=15$; iron deficiency $n=31$ ) versus those with UC (anemia $n=5$; iron deficiency $n=11$ ). This highlights that both iron deficiency and anemia are significantly under-recognized on-going issues in the care of children with IBD and suggests that this remains a problem in this population even several years after diagnosis.

The diagnosis of iron deficiency is currently based on a combination of laboratory measurements: typically mature red blood cell (RBC) measures (low MCV, low MCH, increased RDW), and biochemical markers of iron metabolism (serum Fe, transferrin, transferrin saturation, ferritin, sTfR) $(10,11)$. Of these the most reliable iron biomarkers in healthy children are ferritin and sTfR $(10,11)$. In IBD, however, the diagnosis of iron deficiency is challenging since standard iron biomarkers are altered by inflammatory cytokines independent of changes in iron balance $(7,12)$. Another difficult diagnostic scenario to consider is mild emerging iron deficiency in IBD patients who are responding to therapy and are no longer anemic. Clinically, it would be helpful to be able to identify these changes earlier, to have a sensitive measure of bone marrow response to iron replacement, and to eliminate the delay to see an increase in hemoglobin concentration. A recent review examined the role of reticulocyte markers to differentiate iron deficiency anemia from anemia of chronic disease (16). The authors reported that although there are no studies evaluating $\mathrm{CHr}$ in patients with IBD it could be a reliable index for measuring the response to iron supplementation therapy. Another factor to consider is the cost of different iron biomarkers. Current practices require use of several combinations, with the total average commercial US cost (online supplementary Table S6) depending on which biomarkers are used. Using a single biomarker to effectively diagnose and monitor iron deficiency would substantially reduce the financial burden on the patient/insurance. 
$\mathrm{CHr}$ has been studied in young otherwise healthy (with no inflammation or chronic disease diagnoses) patients to be of use in screening for iron deficiency compared with hemoglobin, ferritin. Ullrich et al. investigated 202 healthy infants aged 9-12 months and reported that $\mathrm{CHr}$ of less than $27.5 \mathrm{pg}$ is a more accurate hematological indicator of iron deficiency (defined as transferrin saturation <10\%) compared with hemoglobin of less than $110 \mathrm{~g} / \mathrm{L}$ (37). Brugnara investigated 210 healthy children (mean age, 2.9 years) and reported $\mathrm{CHr}$ as the strongest predictor of iron deficiency (defined as transferrin saturation <20\%) (38). More recently, Kiudeliene reported that the $\mathrm{CHr}$ is a comparable test with ferritin and transferrin saturation and can be used to detect iron deficiency (diagnosed when at least two of four parameters [ferritin $<12 \mu \mathrm{g} / \mathrm{L}$, transferrin $>3.6 \mathrm{~g} / \mathrm{L}$, transferrin saturation $<10 \%$, sTfR $>1.8 \mathrm{mg}$ / L]were abnormal) in a cohort of 180 healthy children aged 6-24 months (39). An adult study investigated $\mathrm{CHr}$ in 78 adult patients undergoing bone marrow examination as part of an anemia work-up that included benign hematologic disorders and malignancies. They demonstrated $\mathrm{CHr}$ had the highest overall sensitivity and specificity when compared to ferritin, transferrin saturation, and MCV for predicting iron deficiency (defined as lack of stainable iron in bone marrow aspirate) after exclusion of patients with MCV > $100 \mathrm{fL} /$ cell and thalassemia (18).

Our study is the first to date reporting the use of CHr in pediatric IBD patients, and we showed that CHr was correlated with inflammation (as measured by CRP and AGP). Our findings are similar to results reported by Hackeng et al. (40) in a population of adult dialysis patients, showing that $\mathrm{CHr}$ was significantly and inversely related to $\log \mathrm{CRP}$ (rho= $-0.50 ; \mathrm{p}<0.0001)$. We found that $\mathrm{CHr}$ was strongly correlated with both CRP and AGP, possibly due to the high prevalence of both acute and chronic inflammation in our study population (nearly 1 in 2 children had elevated CRP or AGP). Further, as indicated by the high hepcidin values, anemia of chronic disease may have played a role in sequestering iron and thus decreasing CHr levels (41).We showed sTfR to be negatively correlated with inflammation as measured by AGP, which is contrary to prior literature that has shown a positive association between inflammation and $\operatorname{AGP}(42,43)$. In populations with acute inflammation, sTfR has a positive relationship with AGP and hence adjusting it would decrease estimated iron deficiency prevalence. We hypothesize that the opposite correlation in our study may be due to the underlying chronic inflammation in our IBD population that may thus blunt erythropoiesis, and thus sTfR concentrations $(44,45)$.

One of the secondary aims of the study was to determine the diagnostic capacity (sensitivity and specificity) of $\mathrm{CHr}$ as a biomarker of iron deficiency. The following cutoffs for low $\mathrm{CHr}$ to measure iron deficiency had high sensitivity, but moderate specificity: $31 \mathrm{pg}$ unadjusted and $34 \mathrm{pg}$ inflammation-adjusted. Both of these are much higher than other reported cut-offs in the literature: $24 \mathrm{pg}$ in a population of elderly anemic patients (46); $28 \mathrm{pg}$ compared to bone marrow iron stores in adults (18); and $27.5 \mathrm{pg}$ compared to hemoglobin in healthy infants (37). We think that our higher cutoffs both for unadjusted and adjusted $\mathrm{CHr}$ could be related to our not comparing to bone marrow iron as the ROC 'gold standard' as well as the high prevalence of chronic inflammation in our study population.

Adjusted ferritin had the highest sensitivity (79\%) and specificity (90\%) to predict iron deficiency at a threshold value of $20 \mu \mathrm{g} / \mathrm{L}$ and an area under the ROC of 0.83. It is important 
to note however, that both inflammation-adjusted ferritin and sTfR were part of the iron deficiency gold standard for the ROC analysis. Interestingly, contrary to work by Basseri et al. (15), we showed our poorest iron biomarker to be hepcidin (sensitivity $44 \%$, specificity $54 \%$, AUROC 0.51). We had, similar to their group, excluded patients who were receiving iron supplementation or had an MCV >100 fL/cell as potentially having B12 or folate deficiency. However, our sample size was much larger ( $n=62$ versus $n=17)$; we also included patients with both UC and $\mathrm{CD}$ (versus $\mathrm{CD}$ alone) and excluded patients with a recent history of receiving a packed RBC transfusion - these methodological differences could account for our different results. In summary, our analysis showed that all clinically available iron biomarkers are affected by inflammation. One consideration is that different biomarkers reflect different stages and/or types of iron deficiency and will therefore have different prognostic values.

The strengths of the study were as follows: 1) use of regression adjustment of iron biomarkers for use in the ROC analysis; 2) stratified enrollment to ensure a significant proportion of patients with inflammation (and iron deficiency) across the IBD spectrum; 3) evaluation of alternate cut offs for $\mathrm{CHr}$; and 4) measurement of hepcidin which has been proposed to be a measure of anemia of chronic disease. Limitations of the study include the cross-sectional design, preventing causal associations; the relatively small cohort of children; exclusion of individuals with an MCV >100 fL/cell, and the lack of vitamin B12 status measures and menstrual history.

In summary, this study highlights the high prevalence of iron deficiency and anemia in the care of children with IBD. Our evaluation of $\mathrm{CHr}$ as a diagnostic test showed it to be no better than the current standard iron biomarkers. All explored iron biomarkers were affected by inflammation and should be adjusted. A single iron biomarker is unlikely to best predict iron deficiency in pediatric IBD. Iron intervention studies are needed to examine the response of iron biomarkers to iron supplementation in the setting of inflammation and to further evaluate the risk-benefit of iron supplementation in inflamed individuals. Validation of adjusting for the effect of inflammation on iron biomarkers using a regression approach merits further investigation and will help to identify children who may be suitable for therapeutic intervention which in turn may make significant improvements in quality of life.

\section{Supplementary Material}

Refer to Web version on PubMed Central for supplementary material.

\section{Acknowledgments}

This work could not have been completed without the invaluable input of the Kugathasan Lab IBD Dream Team: Kari Aldridge, Corinthian Bryant, Bernadette Martineau, David T. Okou, and Mahadev Prasad. We would also like to thank our clinical team whose help in patient recruitment was critical in the success of this project: Cary G. Sauer, Barbara O. McElhanon, Gail Tenjarla, Walter Ifeadike, Christine Spainhour, Brit Eyster and Lisa Mitchell. Finally, we would also like to acknowledge the support of Janet Gross and Jose Binongo who helped review earlier versions of the proposal.

Grant Support: This work was supported, in part, by grants from the following: National Institutes of Health: 


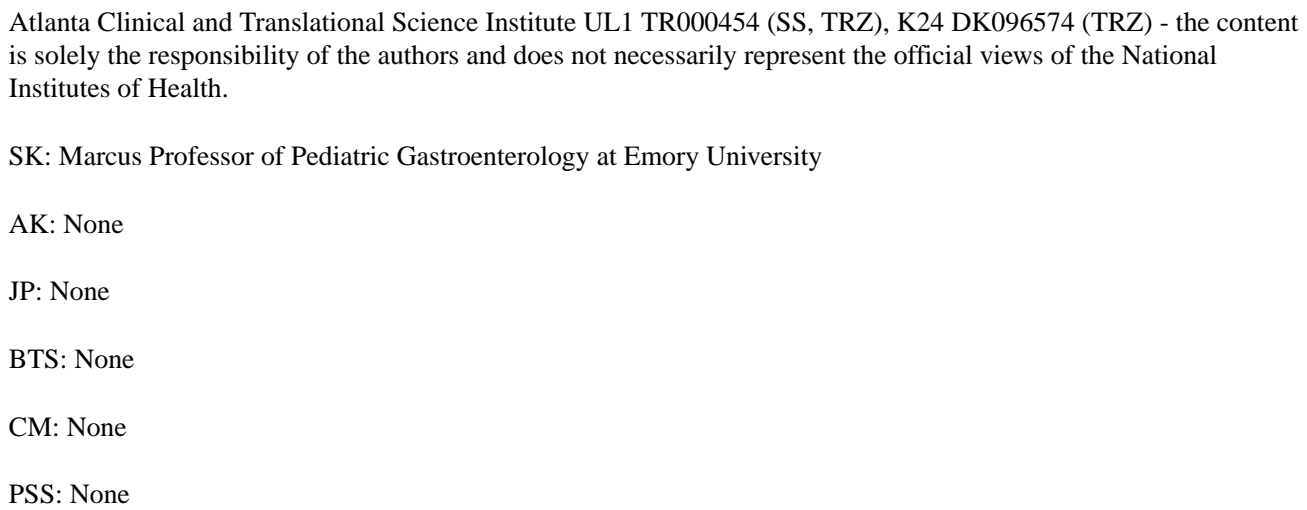

\section{Abbreviations}

CHr hemoglobin content of reticulocytes

ID Iron deficiency

STfR soluble transferrin receptor

CRP C-reactive protein

AGP a 1 acid glycoprotein

ACD anemia of chronic disease

MCV mean cellular volume

RBCs red blood cells

MCV mean corpuscular volume

RDW Red blood cell distribution width

TIBC total iron binding capacity
AA African American
IBD Inflammatory Bowel Disease
UC Ulcerative Colitis
CD Crohn's Disease
PCDAI Pediatric Crohn's Disease Activity Index
PUCAI Pediatric Ulcerative Colitis Activity Index
PGA Physician Global Assessment
BMI Body Mass Index
IU International Units
Kg kilograms 


\section{References}

1. Massironi S, Rossi RE, Cavalcoli FA, Della Valle S, Fraquelli M, Conte D. Nutritional deficiencies in inflammatory bowel disease: therapeutic approaches. Clinical nutrition. 2013; 32(6):904-10. [PubMed: 23602613]

2. Bager P, Befrits R, Wikman O, Lindgren S, Moum B, Hjortswang H, et al. The prevalence of anemia and iron deficiency in IBD outpatients in Scandinavia. Scandinavian journal of gastroenterology. 2011; 46(3):304-9. [PubMed: 21073374]

3. Ganz T. Hepcidin, a key regulator of iron metabolism and mediator of anemia of inflammation. Blood. 2003; 102(3):783-8. [PubMed: 12663437]

4. Camaschella C. Iron-Deficiency Anemia. N Engl J Med. 2015; 373(5):485-6.

5. Ganz T, Nemeth E. Hepcidin and disorders of iron metabolism. Annual review of medicine. 2011; 62:347-60.

6. Michelazzo FB, Oliveira JM, Stefanello J, Luzia LA, Rondo PH. The influence of vitamin A supplementation on iron status. Nutrients. 2013; 5(11):4399-413. [PubMed: 24212089]

7. Oustamanolakis P, Koutroubakis IE, Kouroumalis EA. Diagnosing anemia in inflammatory bowel disease: beyond the established markers. Journal of Crohn's \& colitis. 2011; 5(5):381-91.

8. Lozoff B. Iron deficiency and child development. Food and nutrition bulletin. 2007; 28(4 Suppl):S560-71. [PubMed: 18297894]

9. Bousvaros A, Zurakowski D, Duggan C, Law T, Rifai N, Goldberg NE, et al. Vitamins A and E serum levels in children and young adults with inflammatory bowel disease: effect of disease activity. J Pediatr Gastroenterol Nutr. 1998; 26(2):129-35. [PubMed: 9481625]

10. Baker RD, Greer FR, Committee on Nutrition American Academy of P. Diagnosis and prevention of iron deficiency and iron-deficiency anemia in infants and young children ( $0-3$ years of age). Pediatrics. 2010; 126(5):1040-50. [PubMed: 20923825]

11. WHO/CDC. Assessing the iron status of populations: Report of a Joint World Health Organization/ Centers for Disease Control and Prevention Technical Consultation on the Assessment of Iron Status at the Population Level. Geneva, Switzerland: WHO Library Cataloguing-in-Publication Data; 2007. p. 1-35.

12. Gasche C, Berstad A, Befrits R, Beglinger C, Dignass A, Erichsen K, et al. Guidelines on the diagnosis and management of iron deficiency and anemia in inflammatory bowel diseases. Inflammatory bowel diseases. 2007; 13(12):1545-53. [PubMed: 17985376]

13. Raiten DJ, Sakr Ashour FA, Ross AC, Meydani SN, Dawson HD, Stephensen CB, et al. Inflammation and Nutritional Science for Programs/Policies and Interpretation of Research Evidence (INSPIRE). The Journal of nutrition. 2015; 145(5):1039S-108S. [PubMed: 25833893]

14. Weiss G, Goodnough LT. Anemia of chronic disease. N Engl J Med. 2005; 352(10):1011-23. [PubMed: 15758012]

15. Basseri RJ, Nemeth E, Vassilaki ME, Basseri B, Enayati P, Shaye O, et al. Hepcidin is a key mediator of anemia of inflammation in Crohn's disease. Journal of Crohn's \& colitis. 2013; 7(8):e286-91.

16. Oustamanolakis P, Koutroubakis IE, Messaritakis I, Kefalogiannis G, Niniraki M, Kouroumalis EA. Measurement of reticulocyte and red blood cell indices in the evaluation of anemia in inflammatory bowel disease. Journal of Crohn's \& colitis. 2011; 5(4):295-300.

17. Mast AE, Blinder MA, Dietzen DJ. Reticulocyte hemoglobin content. American journal of hematology. 2008; 83(4):307-10. [PubMed: 18027835]

18. Mast AE, Blinder MA, Lu Q, Flax S, Dietzen DJ. Clinical utility of the reticulocyte hemoglobin content in the diagnosis of iron deficiency. Blood. 2002; 99(4):1489-91. [PubMed: 11830506]

19. Erhardt J. [22nd Sept 2015]. Available from: http://www.nutrisurvey.de/blood_samples/index.htm.

20. Organization WH. Serum ferritin concentrations for the assessment of iron status and iron deficiency in populations. 2011 WHO reference number: WHO/NMH/NHD/MNM/112.

21. Organization WH. Serum transferrin receptor levels for the assessment of iron status and iron deficiency in populations. 2014 WHO reference number: WHO/NMH/NHD/EPG/146. 
22. Organization WH. Serum retinol concentrations for determining the prevalence of vitamin A deficiency in populations. WHO reference number: WHO/NMH/NHD/MNM/113.

23. Organization WH. C-reactive protein concentrations as a marker of inflammation or infection for interpreting biomarkers of micronutrient status. 2014 WHO reference number: WHO/NMH/NHD/EPG/147.

24. Thurnham DI, McCabe LD, Haldar S, Wieringa FT, Northrop-Clewes CA, McCabe GP. Adjusting plasma ferritin concentrations to remove the effects of subclinical inflammation in the assessment of iron deficiency: a meta-analysis. Am J Clin Nutr. 2010; 92(3):546-55. [PubMed: 20610634]

25. Cook JD, Flowers CH, Skikne BS. The quantitative assessment of body iron. Blood. 2003; 101(9): 3359-64. [PubMed: 12521995]

26. McLean E, Cogswell M, Egli I, Wojdyla D, de Benoist B. Worldwide prevalence of anaemia, WHO Vitamin and Mineral Nutrition Information System, 1993-2005. Public health nutrition. 2009; 12(4):444-54. [PubMed: 18498676]

27. Chumlea WC, Schubert CM, Roche AF, Kulin HE, Lee PA, Himes JH, et al. Age at menarche and racial comparisons in US girls. Pediatrics. 2003; 111(1):110-3. [PubMed: 12509562]

28. Levine A, Griffiths A, Markowitz J, Wilson DC, Turner D, Russell RK, et al. Pediatric modification of the Montreal classification for inflammatory bowel disease: the Paris classification. Inflammatory bowel diseases. 2011; 17(6):1314-21. [PubMed: 21560194]

29. Naber AH, de Jong DJ. Assessment of disease activity in inflammatory bowel disease; relevance for clinical trials. Neth J Med. 2003; 61(4):105-10. [PubMed: 12852718]

30. Shepanski MA, Markowitz JE, Mamula P, Hurd LB, Baldassano RN. Is an abbreviated Pediatric Crohn's Disease Activity Index better than the original? J Pediatr Gastroenterol Nutr. 2004; 39(1): 68-72. [PubMed: 15187784]

31. Turner D, Hyams J, Markowitz J, Lerer T, Mack DR, Evans J, et al. Appraisal of the pediatric ulcerative colitis activity index (PUCAI). Inflammatory bowel diseases. 2009; 15(8):1218-23. [PubMed: 19161178]

32. Suchdev PS, Namaste SM, Aaron GJ, Raiten DJ, Brown KH, Flores-Ayala R, et al. Overview of the Biomarkers Reflecting Inflammation and Nutritional Determinants of Anemia (BRINDA) Project. Adv Nutr. 2016; 7(2):349-56. [PubMed: 26980818]

33. Looker AC, Dallman PR, Carroll MD, Gunter EW, Johnson CL. Prevalence of iron deficiency in the United States. Jama. 1997; 277(12):973-6. [PubMed: 9091669]

34. Summary of a report on assessment of the iron nutritional status of the United States population. Expert Scientific Working Group. Am J Clin Nutr. 1985; 42(6):1318-30. [PubMed: 4072962]

35. Knowles J, Thurnham DI, Phengdy B, Houamboun K, Philavong K, Keomoungkhone I, et al. Impact of inflammation on the biomarkers of iron status in a cross-sectional survey of Lao women and children. The British journal of nutrition. 2013; 110(12):2285-97. [PubMed: 23778021]

36. Kasvosve I, Gomo ZA, Nathoo KJ, Matibe P, Mudenge B, Loyevsky M, et al. Association of serum transferrin receptor concentration with markers of inflammation in Zimbabwean children. Clinica chimica acta; international journal of clinical chemistry. 2006; 371(1-2):130-6. [PubMed: 16603144]

37. Ullrich C, Wu A, Armsby C, Rieber S, Wingerter S, Brugnara C, et al. Screening healthy infants for iron deficiency using reticulocyte hemoglobin content. Jama. 2005; 294(8):924-30. [PubMed: 16118382]

38. Brugnara C, Zurakowski D, DiCanzio J, Boyd T, Platt O. Reticulocyte hemoglobin content to diagnose iron deficiency in children. Jama. 1999; 281(23):2225-30. [PubMed: 10376576]

39. Kiudeliene R, Griniute R, Labanauskas L. Prognostic value of reticulocyte hemoglobin content to diagnose iron deficiency in 6-24-month-old children. Medicina. 2008; 44(9):673-7. [PubMed: 18971604]

40. Hackeng CM, Beerenhout CM, Hermans M, Van der Kuy PH, Van der Dussen H, Van DieijenVisser MP, et al. The relationship between reticulocyte hemoglobin content with C-reactive protein and conventional iron parameters in dialysis patients. J Nephrol. 2004; 17(1):107-11. [PubMed: 15151266]

41. Roy CN. Anemia of inflammation. Hematology Am Soc Hematol Educ Program. 2010; 2010:27680. [PubMed: 21239806] 
42. Grant FK, Suchdev PS, Flores-Ayala R, Cole CR, Ramakrishnan U, Ruth LJ, et al. Correcting for inflammation changes estimates of iron deficiency among rural Kenyan preschool children. The Journal of nutrition. 2012; 142(1):105-11. [PubMed: 22157541]

43. Grant FK, Martorell R, Flores-Ayala R, Cole CR, Ruth LJ, Ramakrishnan U, et al. Comparison of indicators of iron deficiency in Kenyan children. Am J Clin Nutr. 2012; 95(5):1231-7. [PubMed: 22456661]

44. Beguin Y. Soluble transferrin receptor for the evaluation of erythropoiesis and iron status. Clinica chimica acta; international journal of clinical chemistry. 2003; 329(1-2):9-22. [PubMed: 12589962]

45. Chiang WC, Tsai TJ, Chen YM, Lin SL, Hsieh BS. Serum soluble transferrin receptor reflects erythropoiesis but not iron availability in erythropoietin-treated chronic hemodialysis patients. Clinical nephrology. 2002; 58(5):363-9. [PubMed: 12425487]

46. Karlsson T. Comparative evaluation of the reticulocyte hemoglobin content assay when screening for iron deficiency in elderly anemic patients. Anemia. 2011; 2011:925907. [PubMed: 21808729] 


\section{SUMMARY BOX}

\section{What is known about this subject?}

- $\quad$ Iron deficiency and anemia affect $50-75 \%$ of IBD patients.

- Iron deficiency is an important contributor to anemia but is difficult to diagnose given the effect of inflammation on iron biomarkers.

- $\quad$ In the setting of inflammation, reticulocyte parameters [(e.g. the hemoglobin content of reticulocytes $(\mathrm{CHr})]$ have been considered promising.

\section{What are the new findings and/or what is the impact on clinical practice?}

- We showed that $\mathrm{CHr}$ is affected by inflammation in a cross-sectional pediatric IBD population.

- $\quad$ Most clinically available iron biomarkers are affected by inflammation and should be adjusted; we suggest using a combination of iron status biomarkers given that there is no single iron status biomarker that predicts iron deficiency in pediatric IBD. 
Patients identified through pediatric inflammatory bowel disease clinics, out-patient infusion clinics, emergency department and the in-patient gastroenterology service at the Children's Healthcare of Atlanta \& Emory University

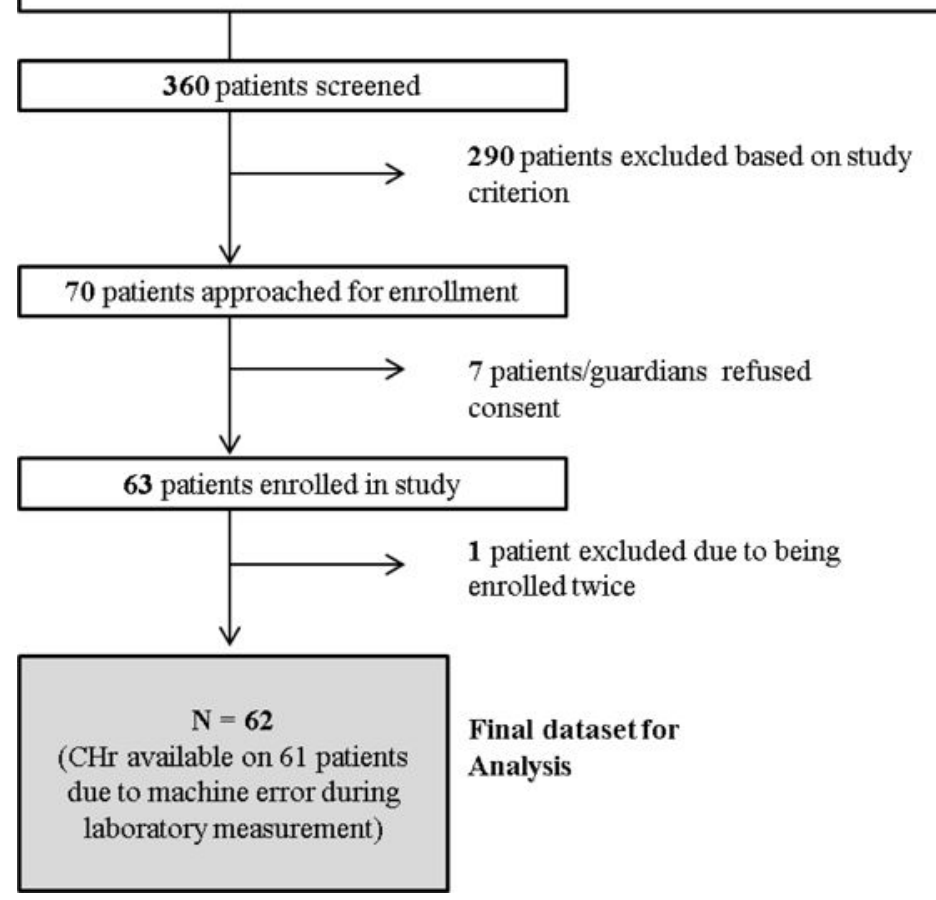

Figure 1.

Flow of Study Participants during six month enrollment period from May 2014 to November $2014(n=62)$ 

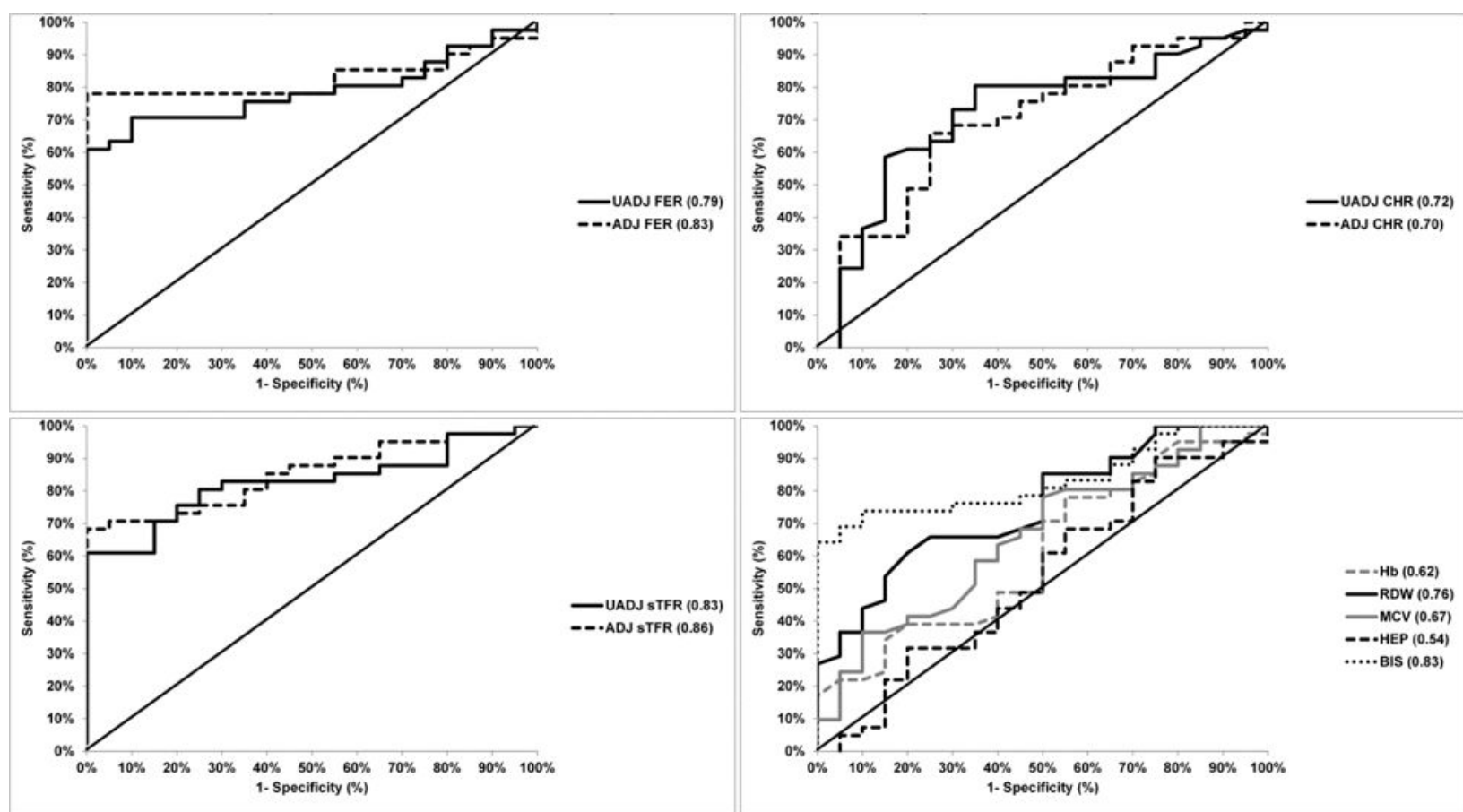

Figure 2. Prognostic values of iron biomarkers (ferritin, reticulocyte hemoglobin concentration, soluble transferrin receptor, hemoglobin, red blood cell distribution width, mean cell volume, hepcidin and Body Iron Stores) according to receiver operating characteristic curve

Note: Lines indicates AUC for each biomarker. Abbreviations: Ferritin= FER; Reticulocyte Hemoglobin Content $=\mathrm{CHr}$; Hemoglobin=Hb; RDW=Red blood Cell Distribution Width; mean cell volume=MCV; HEP=Hepcidin; BIS=Body Iron Stores; soluble transferrin receptor=sTfR; UADJ: unadjusted for inflammation; ADJ: adjusted for inflammation; AUROC: Area under the receiver operating curve 


\section{Table 1}

Iron Deficiency, Inflammation, Anemia \& Vitamin A deficiency in the study population, $n=62^{¥}$

\begin{tabular}{|c|c|}
\hline Biomarkers as categorical variables & Total population $\mathrm{n}(\%)$ \\
\hline \multicolumn{2}{|l|}{ Iron Deficiency (biomarkers not corrected for inflammation) } \\
\hline Low ferritin $<15 \mu \mathrm{g} / \mathrm{L}$ & $20(32 \%)$ \\
\hline Elevated sTfR $>8.3 \mathrm{mg} / \mathrm{L}$ & $25(40 \%)$ \\
\hline Low ferritin or Elevated sTfR & $32(52 \%)$ \\
\hline Low $\mathrm{CHr}<28 \mathrm{pg}$ & $15(25 \%)^{¥}$ \\
\hline Low Body Iron Stores $<0 \mathrm{mg} / \mathrm{kg}$ body weight & $16(26 \%)$ \\
\hline Microcytosis, MCV<75 fL/cell & $7(11 \%)$ \\
\hline Elevated RDW > $14.5 \%$ & $24(39 \%)$ \\
\hline \multicolumn{2}{|l|}{ Inflammation } \\
\hline Elevated $\mathrm{CRP}>5 \mathrm{mg} / \mathrm{L}$ & $25(40 \%)$ \\
\hline Elevated AGP>1.0 g/L & $26(42 \%)$ \\
\hline Elevated CRP or AGP & $30(48 \%)$ \\
\hline Elevated ESR $>15 \mathrm{~mm} / \mathrm{hr}$ & $27(45 \%)$ \\
\hline Low Albumin $<3.5 \mathrm{~g} / \mathrm{dL}$ & $16(26 \%)$ \\
\hline \multicolumn{2}{|l|}{ Iron Deficiency (using regression-correction for inflammation) } \\
\hline Low ferritin $<15 \mu \mathrm{g} / \mathrm{L}$ & $31(50 \%)$ \\
\hline Elevated sTfR $>8.3 \mathrm{mg} / \mathrm{L}$ & $28(45 \%)$ \\
\hline Low ferritin or Elevated TfR & $42(68 \%)$ \\
\hline \multicolumn{2}{|l|}{ Anemia } \\
\hline All & $20(32 \%)$ \\
\hline Females & $9(15 \%)$ \\
\hline Males & $11(18 \%)$ \\
\hline Iron Deficiency Anemia (Iron deficiency not corrected for inflammation) & $15(24 \%)$ \\
\hline Adjusted Iron Deficiency Anemia (Iron deficiency corrected for inflammation) & $17(27 \%)$ \\
\hline \multicolumn{2}{|l|}{ Vitamin A } \\
\hline Deficiency - RBP $<0.7 \mathrm{mmol} / \mathrm{L}$ & $2(3 \%)$ \\
\hline Insufficiency - RBP 0.7 to $<1.0 \mathrm{mmol} / \mathrm{L}$ & $8(13 \%)$ \\
\hline
\end{tabular}

Abbreviations: Reticulocyte Hemoglobin Content=CHr; soluble transferrin receptor=sTfR; C-Reactive Protein=CRP; alpha1-acid glycoprotein=AGP; Retinol Binding Protein (RBP), RDW (Red blood Cell Distribution Width) 


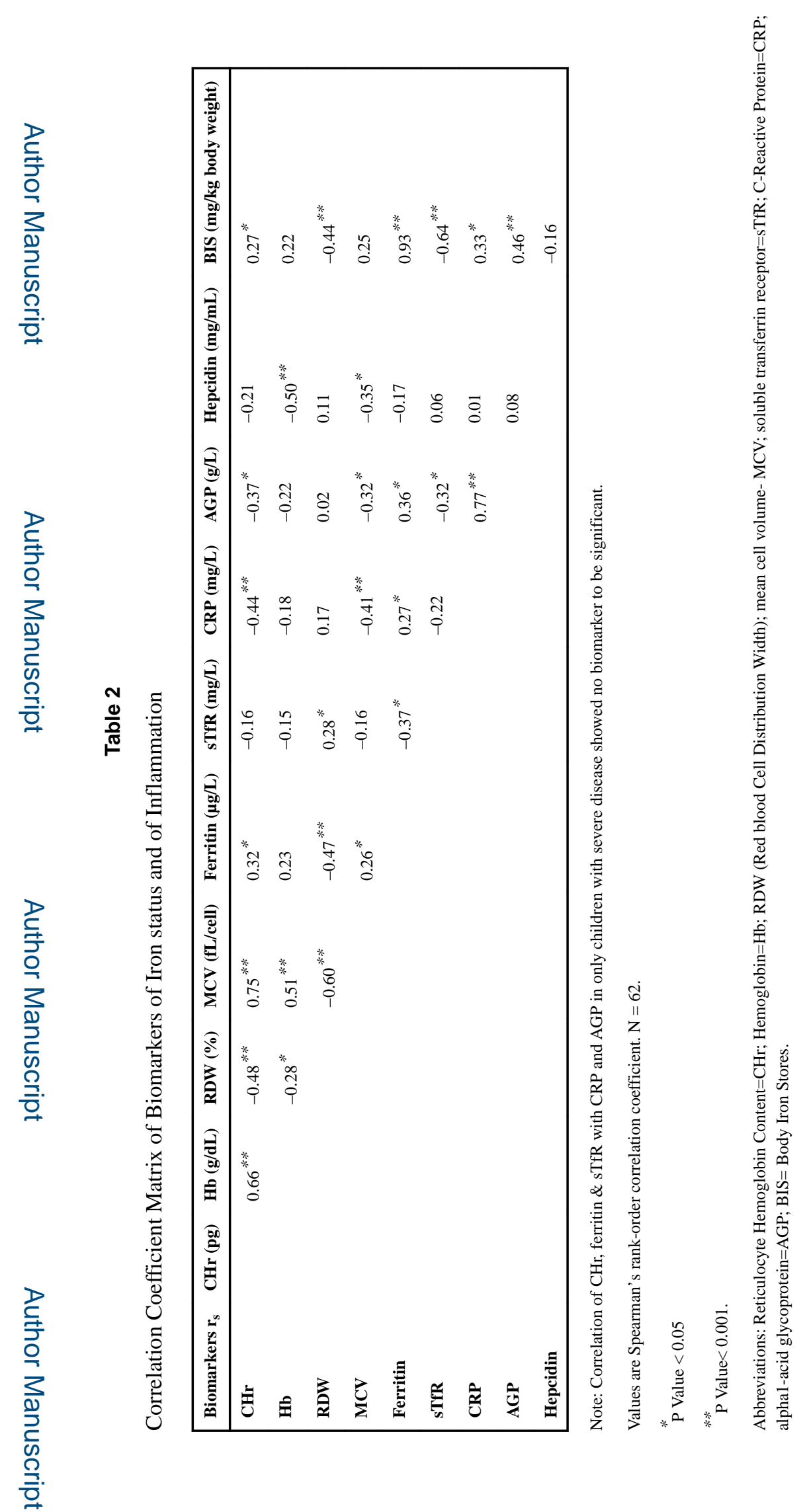

J Pediatr Gastroenterol Nutr. Author manuscript; available in PMC 2018 May 01. 


\section{Table 3}

Optimal prognostic values of different biomarkers for iron deficiency according to receiver operating characteristic curve

\begin{tabular}{|c|c|c|c|c|}
\hline Individual Biomarkers & AUROC & Threshold value & Sensitivity \% & Specificity \% \\
\hline CHr, pg & 0.72 & 31 & $81 \%$ & $60 \%$ \\
\hline $\mathrm{CHr}-$ Adjusted $^{¥}, \mathrm{pg}$ & 0.70 & 34 & $88 \%$ & $30 \%$ \\
\hline $\mathrm{Hb}, \mathrm{g} / \mathrm{dL}$ & 0.62 & 15.1 & $91 \%$ & $25 \%$ \\
\hline RDW, \% & 0.76 & 13.3 & $86 \%$ & $50 \%$ \\
\hline MCV, fL/cell & 0.67 & 86 & $81 \%$ & $40 \%$ \\
\hline Ferritin- Unadjusted, $\mu \mathrm{g} / \mathrm{L}$ & 0.79 & 56 & $88 \%$ & $25 \%$ \\
\hline Ferritin- Adjusted $^{¥}, \mu \mathrm{g} / \mathrm{L}$ & 0.83 & 20 & $79 \%$ & $90 \%$ \\
\hline sTfR- Unadjusted, mg/L & 0.83 & 6.0 & $83 \%$ & $65 \%$ \\
\hline sTfR- Adjusted $^{\#}, \mathbf{m g} / \mathbf{L}$ & 0.86 & 6.6 & $81 \%$ & $60 \%$ \\
\hline Hepcidin, mg/mL & 0.54 & 650 & $45 \%$ & $60 \%$ \\
\hline BIS, mg/kg body weight & 0.83 & 4.3 & $74 \%$ & $75 \%$ \\
\hline
\end{tabular}

Abbreviations: Reticulocyte Hemoglobin Content=CHr; Hemoglobin=Hb; RDW (Red blood Cell Distribution Width); mean cell volume- MCV; soluble transferrin receptor=sTfR; BIS=Body Iron Stores; AUROC: Area under the receiver operating curve.

${ }^{*}$ Regression adjusted for inflammation. 